\title{
The Realization of Artificial Neural Networks in Nonpositional Value for Automated Control Systems
}

\author{
Viktor V. Vasetsky* \\ Military Education and Research Centre of Military-Air Forces \\ «Military-Air Academy \\ Named After Professor N.E. Zhukovsky and Yu.A. Gagarin» \\ 54a Starykh Bolshevikov Str., Voronezh, 394064, Russia
}

Received 03.04.2016, received in revised form 02.09.2017, accepted 30.11.2017

This article raises the problem of using nonpositional number systems, in particular the system of residual classes, which is a parallel system and provides a level parallelism execution of elementary operations in automated control systems.

Keywords: Nonpositional number system, the system of residual classes, artificial neuron, failover neurocomputer.

\section{Реализация искусственных нейронных сетей \\ в непозиционной системе счисления \\ для автоматизированных систем управления}

\section{В.В. Васецкий}

Военный учебно-научный центр Военно-воздушных сил «Военно-воздушная академия имени профессора Н.Е. Жуковского и Ю.А. Гагарина» Россия, 394064, Воронеж, ул. Старых Большевиков, 54 а

В данной статье поднимается проблема использования непозиционных систем счисления, в частности системы остаточных классов, которая является параллельной системой и обеспечивает параллелизм на уровне выполнения элементарных операций в автоматизированных системах управления.

(C) Siberian Federal University. All rights reserved

* Corresponding author E-mail address: vasetsky@inbox.ru 
Ключевые слова: непозиционная система счисления, система остаточных классов, искусственный нейрон, отказоустойчивый нейрокомпьютер.

Одним из методов реализации механизма автоматизированных систем управления в современных условиях является использование нейронных сетей.

Для представления и обработки данных в искусственных нейронных сетях (ИНС) могут быть использованы позиционные и непозиционные системы счисления. Позиционные системы традиционны, и для согласования их с ИНС используются искусственные приемы, которые снижают положительные свойства искусственных нейронных сетей, связанные с параллельными вычислениями. Непозиционные системы счисления, в частности система остаточных классов (СОК), - это параллельные системы, они обеспечивают параллелизм на уровне выполнения элементарных операций, т.е. система остаточных классов выступает естественной основой представления данных в ИНС, служащих их новыми свойствами и возможностями. Этот симбиоз жизненно необходим для исследования ИНС, являющихся базой разработки нейрокомпьютеров, функционирующих в системе остаточных классов. Система в остаточных классах - естественная система счисления для кодирования информации в ИНС, так как математические модели системы остаточных классов и искусственных нейронных сетей являются адекватными моделями [5].

ИНС в СОК - это параллельные взаимосвязанные сети простых элементов, которые предназначены для взаимодействия с объектами реального мира таким же образом, как взаимодействуют биологические нервные системы. Такие сети могут выполнять не только задачи, обладающие естественным параллелизмом, но и некоторые сложные и творческие задачи, реализация которых традиционными методами неэффективна. Для повышения эффективности решения задач возникает необходимость использования ИНС, обладающих свойствами, сходными со свойствами человеческого мозга. При сравнении человеческого мозга с современными компьютерами фон Неймана в плане обработки информации можно заметить, что время переключения искусственных нейронов (несколько миллисекунд) примерно в миллион раз медленнее, чем время переключения элементов современного компьютера, но они имеют в тысячи раз большую соединяемость, чем современный компьютер.

Если входы ИНС сетей с их синаптическими весами отожествить с разрядами чисел, представленных в системе остаточных классов, то ИНС станет натуральным представлением СОК. Соединение естественного параллелизма ИНС и системы остаточных классов создает предпосылки для разработки вычислительных средств с массовым параллелизмом. Кроме того, сочетание свойств ИНС и системы остаточных классов не только реализует массовый параллелизм, но и позволяет с новых позиций разрабатывать отказоустойчивые вычислительные средства. Параллельные вычислительные структуры являются идеальной основой для построения устойчивых к отказам нейрокомпьютерных средств сверхвысокой производительности.

Большинство нейронных алгоритмов включает в себя прежде всего повторяющиеся и регулярные операции. Их можно эффективно отобразить в параллельных структурах.

В ИНС все нейроны работают конкурентно, а на вычисление непосредственно влияет знание, зашифрованное в соединениях сети $[1,4,5]$. Искусственные нейроны объединяются 
в слои. Слой представляет собой совокупность ИН с единым входным сигналом, не имеющих связей между собой. Соотношение, связывающее вход и выход ИН в слое, записывается в следующем виде:

$$
y_{m}=f_{m}\left(\sum_{n=1}^{N} a_{m n} \times x_{m n}+b_{m}\right), \quad m=\overline{1, M}
$$

где $M$ - число ИН в слое; $x=\left(x_{1}, x_{2}, \ldots, x_{N}\right)$ - входной сигнал слоя ИН; $b=\left(b_{1}, b_{2}, \ldots, b_{N}\right)$ - вектор смещения, матрица весовых коэффициентов слоя ИН; $f_{m}\left({ }^{\circ}\right)$ - функция активации $m$-го ИН $A=\left(a_{m n}\right)_{n}^{m}=\overline{1, M}$ в слое; $y_{m}-$ выходной сигнал $m$-го ИН в слое. Слои ИН могут объединяться в нейронную сеть. Соотношение вход-выход $m$-го ИН $j$-го слоя сети таково:

$$
y_{m_{j}}^{j}=f_{m_{j}}^{j}\left(\sum_{n_{j}=1}^{N_{j}} a_{m_{j} n_{j}}^{j} \times x_{n_{j}}^{j}+b_{m_{j}}^{j}\right), \quad m_{j}=\overline{1, M_{j}}, j=\overline{1, J},
$$

остальные символы имеют те же значения, что и в соотношении (1), и относятся к $j$-му слою. Между слоями могут быть установлены связи различного вида. В общем случае выход слоя с номером $j$ подается на вход слоя $j+s$ (рис. 1). Связи называют: последовательными, если $\mathrm{s}=1$; перекрестными, если $s>1$ обратными, если $s<1$.

Если искусственный нейрон (ИН) каждого слоя имеет единую функцию активации $f_{m_{j}}^{j}=f^{j}(\circ) \forall j$, такую ИНС называют однородной $[1,5]$.

Вычисления в искусственных нейронных сетях существенно отличаются от традиционных. В силу высокой распараллеленности их можно рассматривать как коллективное явление. В нейронных сетях нет локальных областей, в которых запоминается конкретная информация. Запоминание информации происходит распределенным образом по всей сети путем модификации весов и порогов. Следствием этого служит то, что ИНС практически индифферентна к потере части вычислительных элементов (нейронов) в процессе работы [5].

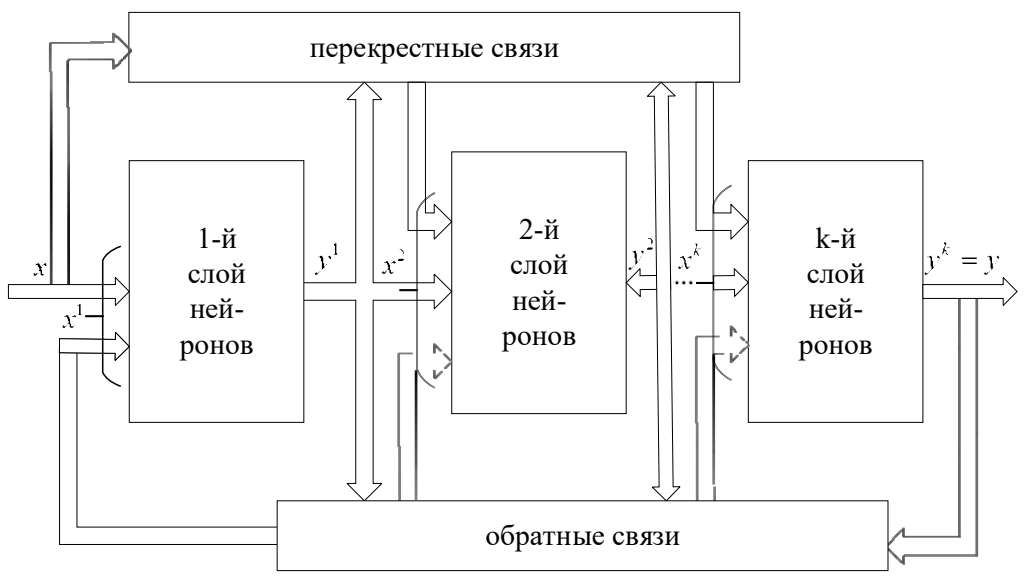

Рис. 1. Многослойная нейронная сеть с перекрестными связями

Fig. 1. Multilayer neural network with cross links 
В СОК число $\mathrm{X}$ кодируется набором остатков $\left(\alpha_{1}, \alpha_{2}, \ldots, \alpha_{n}\right)$ от деления $\mathrm{X}$ на заданные $\left(\alpha_{i}=|X|_{P_{i}},(i=\overline{1, n})\right)$, модули $p_{1}, p_{2}, \ldots, p_{n}$ - основания системы $|X|_{P_{i}}$, символом обозначается элемент множества $|\circ|_{P_{i}}=\{0,1, \ldots, p-1\}$ сравимый с величиной $\mathrm{X}$ по модулю $p_{i}$. Если основания СОК попарно просты $\left(\left(p_{i}, p_{j}\right)=1, i, j=1, n, i \neq j\right)$ то согласно китайской теореме об остатках модулярному коду $\left(\alpha_{1}, \alpha_{2}, \ldots, \alpha_{n}\right)\left(\alpha_{i} \in||_{P_{i}}, i=\overline{1, n}\right)$ соответствует класс вычетов по модулю $P$, задаваемый сравнением

$$
X=\sum_{i=1}^{n} \alpha_{i} B_{i}(\bmod P)=\sum_{i=1}^{n} \alpha_{i} B_{i}-r_{X} P,
$$

где $P=\prod_{i=1}^{n} p_{i}, B_{i}-$ ортогональные базисы СОК; $r_{X}-$ ранг числа Х, показывающий, сколько раз диапазон системы $P$ был превзойден при переходе от представления числа в СОК к его позиционному представлению через систему ортогональных базисов, которые являются константами для заданной системы оснований. Так как сравнения по одному и тому же модулю можно почленно складывать, вычитать и перемножать, то арифметические операции в кольце вычетов по модулю $P$ сводятся к соответствующим операциям над одноименными цифрами модулярного кода операндов по модулям $p_{1}, p_{2}, \ldots, p_{n}$, т.е. выполняется по правилу

$$
|A \circ B|_{P}=\left(\left|\alpha_{1} \circ \beta_{1}\right|_{p_{1}},\left|\alpha_{2} \circ \beta_{2}\right|_{p_{2}}, \ldots,\left|\alpha_{n} \circ \beta_{n}\right|_{p_{n}}\right),
$$

где А и В имеют соответственно модульные коды $\left(\alpha_{1}, \alpha_{2}, \ldots, \alpha_{n}\right)$ и $\left(\beta_{1}, \beta_{2}, \ldots, \beta_{n}\right), \circ \in\{+,-, \times\}$.

По принципу построения СОК каждый остаток $\alpha_{i} \in|\circ|_{P_{i}},(i=\overline{1, k})$ несет информацию обо всем исходном объекте $G$, описываемом информационном кодом $X_{l}(l=\overline{1, P})$ а диапазон представляемых кодовых комбинаций (чисел $\left.X_{l}\right)$ определяется как $(0, P-1)$.

Нейронные вычисления - это проблема для любого уровня последовательных и параллельных ЭВМ. Для каждого вида вычислительной системы нейронные вычисления - проблема супервычислений, проблема достижения большей производительности при тех же реcypcax.

Для эффективных нейровычислений необходимо как можно быстрее выполнять операции перемножения с суммированием результатов, что и обуславливает целесообразность применения СОК в нейросетевых алгоритмах.

С одной стороны, появляется необходимость использования модульных кодовых конструкций в нейрокомпьютерных вычислительных средствах для повышения их отказоустойчивости и ускорения нейрообработки. С другой стороны, в полной мере эффективная реализация СОК может быть достигнута за счет использования адаптивных свойств самих нейронных сетей $[2,3,5]$.

Предпосылкой к созданию нейрокомпьютерных вычислительных средств на основе аппарата системы остаточных классов является семантическое сходство математических моделей нейронных сетей и системы остаточных классов:

1. Математической модели СОК и формального нейрона

$$
X=\sum_{i=1}^{n} \alpha_{i} B_{i}(\bmod P)=\sum_{i=1}^{n} \alpha_{i} B_{i}-r_{X} P \leftrightarrow y=f\left(\sum_{n=1}^{N} x_{n} \times a_{n}+b\right) .
$$

2. Математической модели СОК и перцептронов (простейших многослойных ИНС)

$$
-46-
$$




$$
X=\sum_{i=1}^{n} \alpha_{i} B_{i}(\bmod P)=\sum_{i=1}^{n} \alpha_{i} B_{i}-r_{X} P \leftrightarrow y_{m_{j}}^{j}=f_{m_{j}}^{j}\left(\sum_{n_{j}=1}^{N_{j}} x_{n_{j}}^{j} \times a_{m_{j} n_{j}}^{j}+b_{m_{j}}^{j}\right) .
$$

Таким образом, реализация искусственных нейронных сетей в непозиционной системе счисления для автоматизированных систем управления заключается в реализации арифметики СОК в нейросетевом логическом базисе. Структура алгоритма обработки данных, представленных в системе остаточных классов, как и структура ИНС, обладает естественным параллелизмом, что позволяет использовать ИНС в качестве формального аппарата описания алгоритмов. Кроме того, алгоритмы с ярко выраженным естественным параллелизмом, например обработка сигналов, не используют режима обучения, вместе с тем органически вписываются в нейросетевой логический базис [1]. С этой точки зрения алгоритмы модулярных вычислений соответствуют алгоритмам вычислений с помощью базовых процессорных элементов (искусственных нейронов). По этой причине схемы в СОК адекватны схемам на основе нейросетевого базиса. Искусственные нейронные сети и основные модулярные структуры представляют собой коннекционные устройства, полученные последовательным соединением между собой базовых элементов. Нейронные и модулярные образования будут послойно определены, если задан алгоритм соединения базовых элементов. Аппаратная реализация ИНС, функционирующих в СОК, характерна и для нейроподобных образований, которые обладают максимальным естественным распараллеливанием и служат базой для разработки нового класса вычислительных структур.

\section{Список литературы}

[1] Галушкин А.И. Теория нейронных сетей. М., ИПРЖР, 2000 [Galushkin A.I. The theory of neural networks. M., IRRZHR, 2000 (in Russian)].

[2] Головко В.А. Нейронные сети: обучение, организация и применение. М., ПРЖ. 2001 [Golovko V.A. Neural networks: training, organization and application. M., PRJ. 2001 (in Russian)].

[3] Горбань А.Н. Обучение нейронный сетей. М., СП ПараГраф. 1995 [Gorban A.N. Training neural networks. M., JV ParaGraph. 1995 (in Russian)].

[4] Круглов В.В., Борисов В.В. Искусственные нейронные сети. Теория и практика. М., Горячая линия телеком. 2001 [Kruglov V.V. and Borisov V.V. Artificial neural networks. Theory and practice. M., Hot line by telecom. 2001 (in Russian)].

[5] Нейрокомпьютеры в остаточных классах; ред. А.И. Галушкин, Н.И. Червяков. М., Радиотехника. 2003 [Neurocomputers in the residual classes; Ed. A.I. Galushkin, N.I. Chervyakov. M., Radio engineering. 2003 (in Russian)].

[6] Червяков Н.И. Отказоустойчивые непозиционные процессоры, Управляющие системь и матиныл. 1988. (3) [Chervyakov N.I. Fault-tolerant non-position processors, Control systems and machines. 1988. (3) (in Russian)]. 